\title{
Potassium Titanate Nanoparticles: Effect of Thermal Annealing on the Band Gap Energy
}

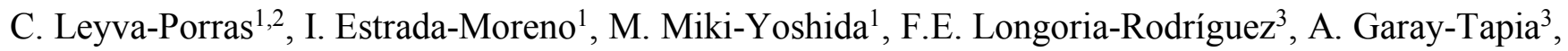
and M. García-Guaderrama².

1. Centro de Investigación en Materiales Avanzados S.C. (CIMAV). Chihuahua, México.

2. Centro de Investigación en Materiales DIP-CUCEI, Universidad de Guadalajara (UdG). Guadalajara, México.

3. Centro de Investigación en Materiales Avanzados S.C. (CIMAV-Mty). Apodaca, México.

Hydrothermal derived titanates nanoparticles (HTN) have received considerable attention because of the onedimensional nanostructure, the simplicity for its synthesis, and band gap energy $\left(\mathrm{E}_{\mathrm{g}}\right)$. Alkali titanates with general formula $\mathrm{A}_{2} \mathrm{Ti}_{n} \mathrm{O}_{2 n+1}$ are commonly synthesized at high concentration of aqueous solution of $\mathrm{NaOH}$ or $\mathrm{KOH}$, and relatively low temperature in the range of $100-250{ }^{\circ} \mathrm{C}$. The microstructure consists of alternate layers of $\mathrm{TiO}_{2}$ octahedra and alkali ions, forming zigzag ribbons. The final microstructure of the titanate polymorph depends on the value of $n$, i.e. layered for $2<n>4$, and tubular for $n>6$. Thus, the chemical composition of the resulting powder plays a major role in the final properties and potential applications of the material. The relative position of $\mathrm{E}_{\mathrm{g}}$ with respect to the hydrogen electrode is the main disadvantage, because the material is active only under ultraviolet (UV) illumination. Thus, it is desirable that the HTN be activated under visible light conditions. Several works have reported the effect of the annealing temperature on the evolution of the crystalline phases, and microstructure of HTN, but few have reported the changes in the $E_{g}$. Amano et al. attributed the decrease in the $E_{g}$ to the change of the chemical composition [1]. Allen et al. involved the formation of microstructural defects in the reduction of $\mathrm{E}_{\mathrm{g}}$ [2]. Therefore, in this work is presented the microstructural characterization of the HTN particles annealed at different temperatures, and its relation with the changes in the band gap energy.

Potassium titanates were synthesized via the hydrothermal reaction of $\mathrm{TiO}_{2}$ anatase nanoparticles with $10 \mathrm{M}$ $\mathrm{KOH}$ solution, at $170{ }^{\circ} \mathrm{C}$ by 24 hours. Collected powders were thermally annealed from $500-1100{ }^{\circ} \mathrm{C}$ by 2 hours. The evolution of the particles with the annealing temperature was followed in a HRTEM JEOL JEM$2200 \mathrm{FS}+\mathrm{Cs}$.

Figure 1 shows the increase in the particle size in the range of 10-200 nm, and the variation of the morphology, from needle-like particles into faceted particles. Figure 2 shows HRTEM images, observing the typical layered arrangement displayed by the HTN. The interplanar distances decreased with the annealing temperature, indicating the phase change. This was confirmed by the selected area electron diffraction patterns showed in the insets of figure 2. The evolution of the phases was the transformation of layered titanates into anatase, and anatase into rutile. However, a mixture of $\mathrm{HTN}$ and $\mathrm{TiO}_{2}$ was observed at temperatures above $700{ }^{\circ} \mathrm{C}$. Figure 3 shows the determined $E_{g}$ plotted against the annealing temperature; overall, $E_{g}$ decreased from 3.43-2.95 eV. This phenomenon can be explained in terms of the coupling between the corresponding conduction bands, and valence bands of the $\mathrm{HTN}$, and $\mathrm{TiO}_{2}$, which yields in the formation of a heterojunction. A similar behavior was observed by Scanlon et al. who explained that the great performance displayed by the Degussa P25 in photocatalysis and energy conversion applications, was caused by the coupling of the valence and conduction bands of the anatase and rutile phases [3].

These results suggest that the decrease in the band energy observed macroscopically was caused by the synergistic effect of a heterojunction produced during the intimate contact between the different crystalline phases of potassium titanates, anatase, and rutile. Clearly, this study contributes to the understanding of the 
phase transformation of potassium titanates, and the improvement in the properties of absorption, and conversion of light into energy.

References:

[1] F. Amano, et al. Applied Catalysis B: Environmental 89 (2009), p. 583.

[2] M.R. Allen, et al. Chemistry of Materials, 22 (2010), p. 1220.

[3] D.O. Scanlon, et al. Nature materials, 12 (2013), p. 798.
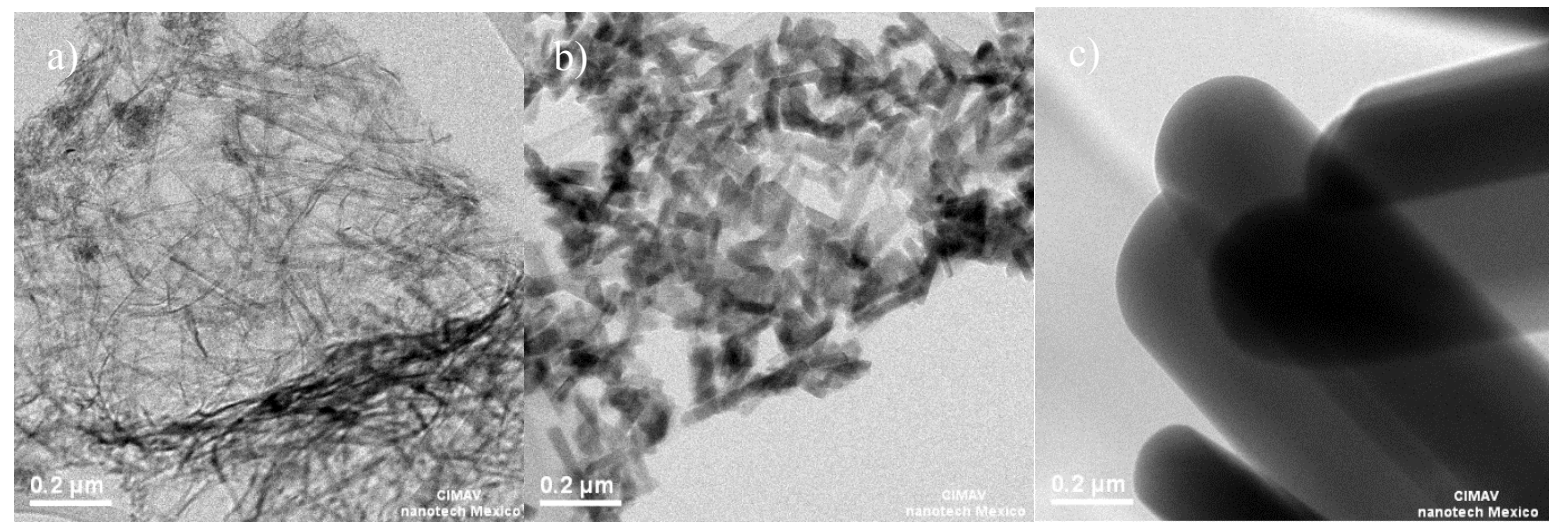

Figure 1. Low magnification TEM images of HTN: a) without thermal annealed, b) annealed at $800{ }^{\circ} \mathrm{C}$, and c) annealed at $1100^{\circ} \mathrm{C}$.

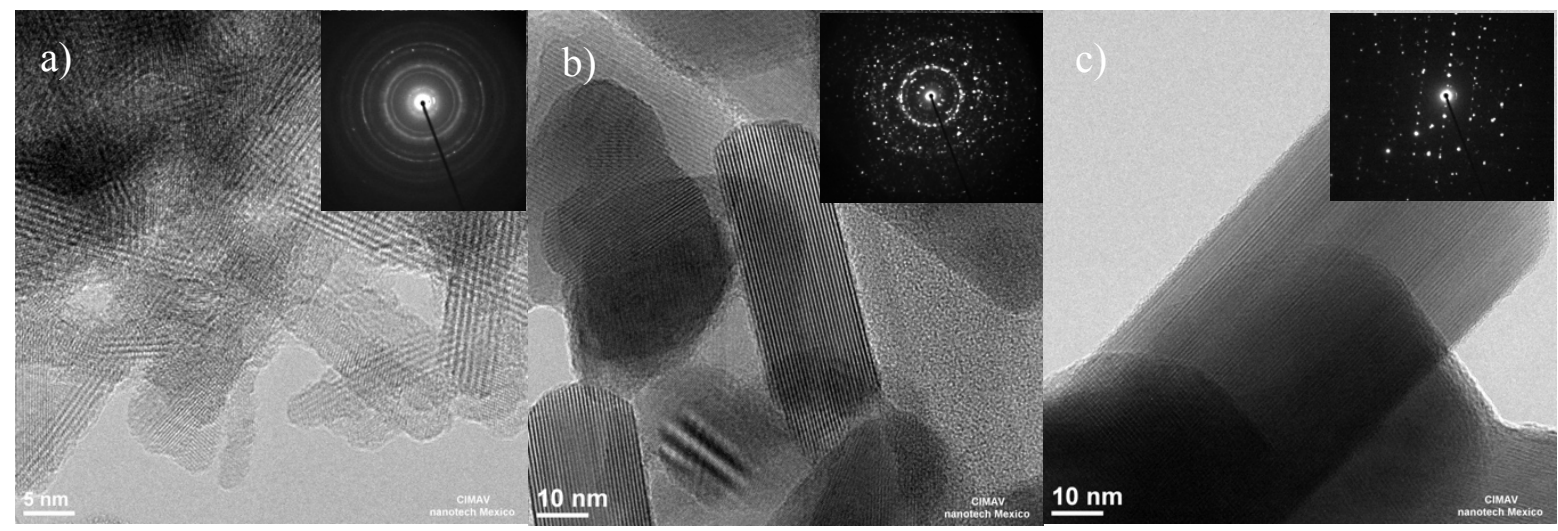

Figure 2. HRTEM images of HTN: a) without thermal annealed, b) annealed at $800{ }^{\circ} \mathrm{C}$, and c) annealed at $1100{ }^{\circ} \mathrm{C}$.

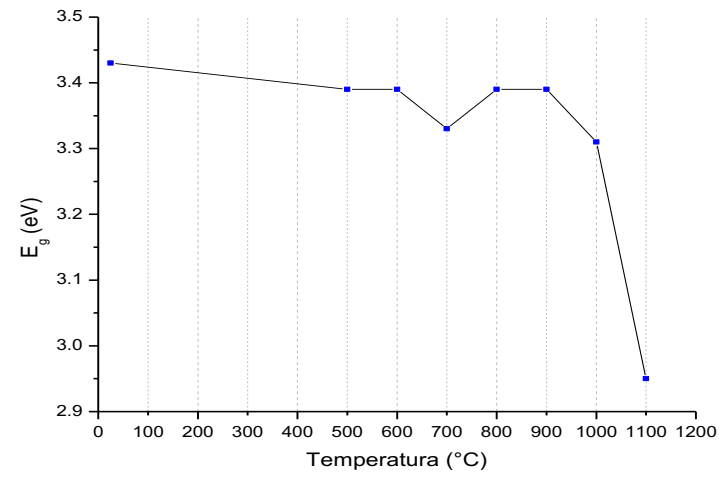

Figure 3. Effect of annealing temperature on the band gap energy. 